\title{
A pilot study on the use of letrozole with either misoprostol or mifepristone for termination of pregnancy up to 63 days
}

\author{
Vivian Chi Yan Lee, Oi Sang Tang, Ernest Hung Yu Ng, \\ William Shu Biu Yeung, Pak Chung Ho. \\ Department of Obstetrics \& Gynaecology, the University of Hong Kong, \\ Hong Kong Special Administrative Region, People's Republic of China.
}

Corresponding author: Pak Chung Ho.

Department of Obstetrics and Gynaecology, The University of Hong Kong, Queen Mary Hospital, Pokfulam Road, Hong Kong Telephone: $\quad 852-28553400$

Fax: $852-28175374$

E-mail: $\quad$ pcho@hkusua.hku.hk 


\begin{abstract}
Background: Letrozole is a third-generation selective aromatase inhibitor. Animal data suggested that it might be useful in medical abortion. We performed two pilot studies to assess the feasibility of using letrozole in combination with either mifepristone or misoprostol for termination of pregnancy up to 63 days.
\end{abstract}

Study design: We recruited 40 subjects who requested legal termination of pregnancies up to 63 days. Medical abortion was performed with letrozole $7.5 \mathrm{mg}$ daily for two days followed by 800 mcg vaginal misoprostol in 20 subjects and letrozole $7.5 \mathrm{mg}$ combined with $200 \mathrm{mg}$ mifepristone in another 20 subjects.

Results: The mean induction-to-abortion interval of the regimen of letrozole and misoprostol was 9.1 hours (median 7.9 hours, range 2.7-23.6 hours). The complete abortion rate was $80 \%$ (95\%CI: $56.3-94.3 \%$ ). For those with gestation $\leq 49$ days, the complete abortion rate was $87.5 \%$ (14/16; 95\%CI: 61.7-98.5\%). The mean induction-to-abortion interval of letrozole combined with mifepristone was 90.1 hours (median 93.4 hours, range 66.0-121.2 hours). The complete abortion rate was $71.4 \%$ (95\% CI: 47.8-88.7\%).

Conclusion: These preliminary results suggest that a regimen of letrozole and misoprostol may be useful in medical abortion, but the combination with mifepristone is less effective and takes longer. Randomized studies comparing letrozole and misoprostol to misoprostol alone are warranted.

Keywords: first trimester medical abortions; letrozole; mifepristone; misoprostol 


\section{Introduction}

It was estimated that there were 42 million induced abortions in 2003, compared with 46 million in 1995[1]. Approximately $90 \%$ of abortions were performed in the first trimester [2, 3]. Induced abortions can be performed by surgical or medical methods. The recommended regimen for medical abortion in the first trimester is the sequential use of mifepristone (RU 486) followed one to three days later by a prostaglandin analogue. Mifepristone is an anti-progesterone that can increase the sensitivity of the uterus to prostaglandins. However, the complete abortion rate of using mifepristone alone was only $60-80 \%$ for termination of early pregnancy less than 6 weeks $[4,5]$. This complete abortion rate is too low to be acceptable especially when it is compared to surgical abortion which has a complete abortion rate of more than $95 \%$. The complete abortion rate with prostaglandins alone was over $90 \%$ when repeated doses were given or when the misoprostol tablets were soaked with normal saline $[6,7]$. The complete abortion rate could be increased to $95 \%$ when the administration of mifepristone was followed by a suitable

prostaglandin analogue $[8,9]$. Therefore, the sequential regimen of mifepristone and a prostaglandin analogue is now accepted as the method of choice in medical abortion in the first trimester. However, the widespread use of this sequential regimen of mifepristone and a prostaglandin analogue is limited by the fact that mifepristone is expensive and is not available in many countries. There is a need to find a cheaper and easily available alternative.

Letrozole is a third-generation aromatase inhibitor which is used to treat estrogen-dependent breast cancer. Aromatase, an enzyme of the cytochrome P450 superfamily and the product of CY195 gene, is expressed in the placenta and ovarian granulosa cells [10]. In premenopausal 
women, the use of aromatase inhibitors leads to an increase in gonadotropin secretion because of the reduced feedback of estrogen to the hypothalamus and pituitary which is the underlying mechanism of its use in ovulation induction [11-14]. Third-generation aromatase inhibitors have specific actions at clinical doses with no effect on basal levels of cortisol and aldosterone [15-17]. Both estrogen and progesterone are important hormones for the maintenance of pregnancy. The role of estrogen in pregnancy maintenance in primates was shown by Albrecht et al. [18], who demonstrated that after administration of an aromatase inhibitor, maternal estrogen synthesis was suppressed and estradiol concentration was substantially decreased. Half of the treated baboons had a miscarriage. In contrast, all the baboons treated concomitantly with estradiol and aromatase inhibitor maintained their pregnancies. Serum progesterone concentrations were not significantly different throughout the experimental period. They concluded that estrogen, acting directly, indirectly, or both through a factor or factors other than progesterone, plays a critical physiologic role in the maintenance of primate pregnancy.

Shi et al.[19] demonstrated that when low doses of mifepristone were combined with low doses aromatase inhibitors, both had no effect when given alone, a tremendous synergistic effect was seen in the mouse, the rat and the guinea pig. Abortion rates were up to $100 \%$. A complete evacuation of the uterus occurred soon after the treatment and the duration of bleeding was surprisingly short.

In order to evaluate the role of letrozole use for first trimester abortions in humans, we performed two pilot studies to assess the feasibility of the following two regimens for termination of pregnancy up to 63 days: 
1. letrozole $7.5 \mathrm{mg}$ daily for two days followed by $800 \mathrm{mcg}$ vaginal misoprostol and

2. letrozole $7.5 \mathrm{mg}$ combined with $200 \mathrm{mg}$ mifepristone.

\section{Materials and methods}

Women requesting legal termination of pregnancy at a gestational age less than or equal to 63 days at Queen Mary Hospital were recruited for these two pilot studies. Ethical approval was obtained from the University of Hong Kong, Hospital Authority Hong Kong West Cluster Institutional Review Board. The trial was registered; trial number HKCTR146 at www.hkuclinicaltrials.com. Information sheets were given to eligible subjects and written consent was obtained from all subjects after extensive counseling.

The inclusion criteria were: good general health; older than the age of legal consent (i.e. $>18$

years old); requesting medical abortion and eligible for legal abortion; on Day 1 of the study (day of letrozole administration) the duration of pregnancy was not more than 63 days confirmed by transvaginal ultrasound (crown rump length of less than $22 \mathrm{~mm}$ ); willing to use contraception other than hormonal or intrauterine contraception until the first menses after termination of pregnancy; agreement by the woman to undergo termination of pregnancy with a surgical method if treatment fails; willing and able to participate after the study had been explained; hemoglobin higher than $10 \mathrm{~g} / \mathrm{L}$. The exclusion criteria included: history or evidence of adrenal pathology, steroid-dependent cancer, porphyria, diastolic pressure over $95 \mathrm{~mm} \mathrm{Hg}$, bronchial asthma, arterial hypotension; history or evidence of thromboembolism, severe or recurrent liver disease or pruritus of pregnancy; regular use of prescription drugs before admission to the study; 
presence of an intrauterine device; breastfeeding; heavy smoker of more than 20 cigarettes per day; any abnormal values in pre-treatment blood tests. The conditions where women could be excluded from a secondary analysis included: the presence of an acute illness of any nature during the treatment period; the use of drugs other than those prescribed by the investigator for the treatment of possible therapy-related side effects; any violation of the study protocol; essential data missing from the participants' records making it impossible to judge treatment outcome.

Women requesting termination of pregnancy at our clinics were screened by a research nurse. If they fulfilled the criteria for inclusion, the nurse explained the project to the women and gave them the information sheets. When they agreed to participate in the study, they were seen by one of the doctors on the research team. After a detailed history and a full physical examination, an ultrasound examination was performed to confirm an intrauterine pregnancy and the gestational age. Complete blood count, renal and liver function, serum progesterone, estradiol and cortisol concentrations were checked. A written consent was obtained from each subject.

Subjects admitted to the study were given a diary to record days and amount of vaginal bleeding and side effects. The diary also gave the dates of the required clinic visits starting with the stay in the hospital after the start of the treatment.

\subsection{Treatment regimen}

\subsubsection{Letrozole and misoprostol regimen}

Subjects took the first dose of letrozole under the supervision of the project nurse and then took 
the second dose herself on Day 2. They were admitted to the hospital on Day 3 for the administration of vaginal misoprostol. Subjects stayed in the hospital after the administration of misoprostol for $4 \mathrm{~h}$. Side effects, bleeding data and time of expulsion were recorded. They were discharged after the 4-h observation period if the vaginal bleeding was not heavy and abdominal pain was not severe. The first follow-up visit took place one week after the start of treatment (Day 7) during which a pelvic ultrasound was performed. The second follow-up visit was on Day 43 and they were followed up until the return of the first menstruation after abortion, if needed. A blood sample was taken for hemoglobin level, liver and renal function tests, serum estradiol, progesterone and cortisol concentrations on Day 3 (on the day of hospital admission before the administration of misoprostol), Day 7 and Day 43.

\subsubsection{Letrozole and mifepristone regimen}

Subjects received a single oral dose of $7.5 \mathrm{mg}$ letrozole and $200 \mathrm{mg}$ of oral mifepristone on Day 1 under the supervision of the project nurse followed by a single dose of $7.5 \mathrm{mg}$ letrozole on Day

2. They were kept under observation in the hospital after the administration of letrozole and mifepristone for 2 days. Subjects were allowed to go home after the observation period if there was no heavy bleeding or severe abdominal pain. The outcomes of the treatment were assessed at follow-up visits on Days 7 and 43 of the study, as above. Hormonal and hematological investigations were carried out before treatment, on Day 3, Day 7 and Day 43 follow-up visits.

\subsection{Assessment of efficacy}

The primary treatment outcome of both pilot studies was complete abortion and the secondary outcomes included the induction-to-abortion interval and missed abortion. 
In subjects with ongoing pregnancies on Day 7 follow-up visit, surgical method was used. Otherwise, no further action was taken unless there was heavy vaginal bleeding. In these women the final judgment on the outcome of therapy was made at the follow-up on Days 43 . If no emergency or elective curettage was necessary during the period up to the first menstruation, the outcome of treatment was classified as "complete abortion". The remaining cases in whom curettage was done were classified as "incomplete abortion" and tissues removed by curettage were sent for histological examination.

\subsection{Assessment of safety}

The amount of vaginal bleeding, side effects and vital signs during the hospital stay were recorded by the nursing staff. Subjects enrolled in the studies received a diary to record vaginal bleeding and side effects they experienced. The research nurse clarified with the subjects the nature and details of side effects on follow-up visits.

\subsection{Hormone assays}

Blood samples were processed by centrifuge. The serum levels of estradiol and progesterone were measured by immunoassay system from Beckman Coulter (Fullerton, CA). The sensitivity of these assays is $73 \mathrm{pmol} / \mathrm{L}$ for estradiol and $0.25 \mathrm{nmol} / \mathrm{L}$ for progesterone; the intra-assay coefficients of variation (CVs) are $4.0 \%$ and $4.4 \%$, and the inter-assay CVs are $5.0 \%$ and 3.6\%

for estradiol and progesterone, respectively. The serum level of cortisol was measured by immunoassay system from Siemens Medical Solutions, Diagnostics Ltd. (HK, China). The sensitivity of the assay is $5.6 \mathrm{nmol} / \mathrm{L}$; the intra-assay and inter-assay CVs are $3.69 \%$ and $5.45 \%$. 


\subsection{Statistical analysis}

Treatment outcomes included complete abortion, incomplete abortion, failed attempted abortion (continuing live pregnancy), or undetermined outcome. Crude proportions for each outcome were estimated. Crude proportions of failure to have a complete abortion were calculated, with exact confidence intervals based on the Binomial distribution. A total of 20 subjects were enrolled in each pilot study.

\section{Results}

\subsection{Letrozole and misoprostol regimen}

The demographic data including age, height, body weight and gestational age are presented in Table 1. The mean interval between misoprostol administration and abortion was $9.1 \mathrm{~h}$ (median $7.9 \mathrm{~h}$, range $2.7-23.6 \mathrm{~h}$ ). The mean length of vaginal bleeding was 20.1 days (median 12.0, range 7-45 days). The complete abortion rate was $80.0 \%$ (16/20; 95\% CI: 56.3-94.3\%). Four subjects had an ongoing pregnancy requiring surgical treatment. One subject failed to return for follow-up on Day 43 (Table 2). In a subgroup analysis of those with gestation $\leq 49$ days, the complete abortion rate was $87.5 \%(14 / 16 ; 95 \%$ CI: $61.7-98.5 \%)$.

The common side effects after letrozole pretreatment were fatigue (30\%) and nausea (25\%) while after misoprostol administration, lower abdominal pain (65\%) and nausea (35\%) were the common side effects. Most of the side effects, such as nausea, vomiting, fatigue, dizziness and headache, were already present during the pregnancy before administration of treatment, and they persisted after letrozole pretreatment and misoprostol administration. There was one patient with fever of $\geq 38^{\circ} \mathrm{C}$ during misoprostol administration. Almost all side effects subsided on the 
second follow-up visit (on D43), except three patients complaining of lower abdominal pain with no significant gynecological causes found (Table 3).

The hemoglobin level remained stable throughout the study period (Fig 1). Renal and liver function tests were all normal. The median serum estradiol concentration showed a significant drop from enrolment to Day 3 after letrozole ingestion,and continued to drop until Day 7 visit with an increase at Day 43. The median serum progesterone concentration remained steady from enrolment to Day 3 after letrozole ingestion; it dropped on Day 7 follow-up visit and continued to drop until Day 43. There was no significant change in the cortisol level throughout the followup period.

\subsection{Letrozole and mifepristone regimen}

The demographic data were shown in Table 1. The mean interval between first dose of letrozole administration and the abortion was $90.1 \mathrm{~h}$ (median $93.4 \mathrm{~h}$, range 66.0-121.2 h). The mean length of vaginal bleeding was 21.0 days (median 13.5 days, range 5-66 days). The complete abortion rate was $71.4 \%(15 / 21 ; 95 \% \mathrm{CI}: 47.8-88.7 \%)$. Five (25\%) subjects had an ongoing pregnancy requiring surgical treatment. One subject withdrew from the study and she had suction evacuation done by a private gynecologist because she refused to wait until the follow-up day (Table 2). In the subgroup analysis on subjects of gestation $\leq 49$ days, the complete abortion rate was $83.3 \%(10 / 12 ; 95 \% \mathrm{CI}: 51.6-97.7 \%)$ while there was only one ongoing pregnancy in this group of patients and one subject withdrew. 
The common side effects after letrozole and mifepristone were nausea (42.9\%) and lower abdominal pain (38.1\%). During the first follow-up on Day 7 , lower abdominal pain $(84.2 \%)$ and fatigue (42.1\%) were the most common side effects. All side effects subsided on the second follow-up visit on Day 43 (Table 3).

The hemoglobin level remained stable throughout the study period (Fig 2). Renal and liver function tests were all normal. The median serum estradiol concentration showed a significant drop from enrolment to Day 3 after letrozole ingestion, and remained steady onwards to Day 7 , which increased on Day 43. The median serum progesterone concentration remained steady from enrolment to Day 3 after letrozole and mifepristone ingestion; it dropped on Day 7 follow-up and continued to drop until Day 43. The median of serum cortisol concentration rose from Day 1 to Day 3 while there was a drop on Day 7 and remained steady until Day 43 follow-up. 


\section{Discussion}

The sequential regimen of mifepristone followed by a suitable prostaglandin such as vaginal misoprostol is the regimen of choice for medical abortion in the first trimester because it is more effective than the use of either mifepristone or misoprostol alone. However, mifepristone is not available in many parts of the world, including Hong Kong. Therefore, we conducted this pilot study to assess the feasibility of using letrozole to improve the efficacy of misoprostol in termination of first trimester pregnancy. To the best of our knowledge, this is the first study using the regimens of letrozole and misoprostol and the combined letrozole and mifepristone in first trimester medical abortions.

The regimen of 2 days of letrozole $7.5 \mathrm{mg}$ daily combined with vaginal misoprostol $800 \mathrm{mcg}$ was associated with a complete abortion rate of $80 \%$ in subjects with gestation less than 63 days. In subjects with gestation less than 49 days, the complete abortion rate was higher $(87.5 \%)$. These were much lower than the complete abortion rates of $93-95 \%$ using the sequential regimen of mifepristone and vaginal misoprostol with intervals of 1-3 days between mifepristone and misoprostol $[3,20]$. However, mifepristone is not available in many countries where misoprostol alone has to be used for medical abortion. Our group showed that by using repeated doses (up to 3) of vaginal misoprostol alone, the success rate was only $65 \%$ when using dry misoprostol and $85 \%$ when using vaginal misoprostol moistened with water [21]. The complete abortion rate with a single dose of vaginal misoprostol moistened with water was only $72 \%$ in an early study [22], while in a recent randomised trial revealed the complete abortion rate with a single dose of vaginal misoprostol moistened with saline of $94.2 \%$ in pregnancy up to 49 days of gestation [7]. 
Therefore, the success rate in this pilot study (where a single dose of dry vaginal misoprostol was used) seems to be promising when compared with those in most studies using a single dose of vaginal misoprostol or dry tablets of vaginal misoprostol. Further randomized controlled studies are warranted to confirm our findings in termination of first trimester pregnancies.

The combined letrozole and mifepristone regimen was based on the exciting result that the abortion rate would be $100 \%$ with the combination of low doses of mifepristone with aromatase inhibitors in mice, indicating a tremendous synergistic effect [19]. However, it seemed that it was not the case for humans as the complete abortion rate was only $71.4 \%$ which was lower than the mifepristone-misoprostol or even letrozole-misoprostol regimens. The dramatic synergistic effect of mifepristone and letrozole seen in mice was not seen in humans. We are not sure whether it is due to improper dosage or species difference.

Both pilot studies showed a significant drop of serum estradiol concentration after letrozole ingestion on Day 3 without any change in the serum progesterone concentration, which was compatible with the previous work on the effect of letrozole on hormonal levels [23]. The cortisol concentration was not altered in the letrozole and misoprostol regimen, indicating that letrozole does not have any significant effects on the cortisol levels. The significant increase in cortisol concentration in the letrozole and mifepristone regimen from Day 1 to Day 3 was due to the antiglucocorticoid action of mifepristone at the receptor level. We did not encounter any serious complications in this pilot study and there was no significant change in hemoglobin, liver and renal function tests, indicating that both regimens are probably safe but a larger series is necessary to confirm their safety. 
Letrozole has been reported on the use in treatment of breast cancer and ovulation induction.

There was no report on its use in medical abortion. The results of this pilot study suggested that the efficacy of using $7.5 \mathrm{mg}$ letrozole for 2 days before $800 \mathrm{mcg}$ vaginal misoprostol in medical abortion might improve the efficacy of vaginal misoprostol in termination of pregnancy less than 63 days, although it is less effective than mifepristone. We postulated that the effect of letrozole might be mediated through the placentae or corpus luteum in which suppressed estrogen biosynthesis may be detrimental to their functions. However, further studies were required to elucidate the underlying mechanism of letrozole in medical abortion. Our findings require properly conducted prospective randomized trials to confirm the results. In view of unavailability of mifepristone in some parts of the world, the exploration of another agent is vital in order to provide safe and effective abortion services. However, the optimal dosage and duration of administration remains unknown and further prospective trials are required to answer these questions. We postulate that the use of a higher dosage of letrozole may be beneficial, especially in a later gestation, of which the estradiol concentration is high than that of early gestation.

\section{References}

[1] Sedgh G, Henshaw S, Singh S, Åhman E, Shah IH. Induced abortion: estimated rates and trends worldwide. Lancet 2007;370:1338-45.

[2] Gamble SB, Strauss LT, Parker WY, Cook DA, Zane SB, Hamdan S. Abortion surveillance - United States, 2005. MMWR 2008;57:1-32.

[3] El-Refaey H, Rajasekar D, Abdalla M, Calder L, Templeton A. Induction of abortion with mifepristone (RU 486) and oral or vaginal misoprostol. N Engl J Med 1995;332:983-7.

[4] Couzinet B, Le Strat N, Ulmann A, Baulieu E, Schaison G. Termination of early pregnancy by the progesterone antagonist RU 486 (Mifepristone). N Engl J Med 1986;315:1565-70.

[5] Kovacs L, Sas M, Resch BA et al. Termination of very early pregnancy by RU 486--an antiprogestational compound. Contraception 1984;29(5):399-410.

[6] Fekih M, Fathallah K, Regaya LB, Bouguizane S, Chaieb A, Bibi M, et al. Sublingual misoprostol for first trimester termination of pregnancy. Int J Gynecol Obstet 2010;109:6770. 
[7] Prasad S, Kumar A, Divya A. Early termination of pregnancy by single-dose $800 \mathrm{mcg}$ misoprostol compared with surgical evacuation. Fertil Steril 2009;91:28-31.

[8] Bygdeman M, Swahn ML. Progesterone receptor blockage: Effect on uterine contractility and early pregnancy. Contraception 1985;32:45-51.

[9] Cameron IT, Michie AF, Baird DT. Therapeutic abortion in early pregnancy with antiprogestogen RU486 alone or in combination with prostaglandin analogue (gemeprost). Contraception 1986;34:459-68.

[10] Cole P, Robinson C. Mechanism and inhibition of cytochrome P-450 aromatase. J Med Chem 1990;33:2933-42.

[11] Mitwally MF, Casper RF. Aromatase Inhibition: A Novel Method of Ovulation Induction in Women With Polycystic Ovary Syndrome. Reprod Technol 2001;10:244-7.

[12] Mitwally MF, Casper RF. Single-dose administration of an aromatase inhibitor for ovarian stimulation. Fertil Steril 2005;83:229-31.

[13] Holzer H, Casper R, Tulandi T. A new era in ovulation induction. Fertil Steril 2006;85:277-84.

[14] Badawy A, Metwally M, Fawzy M. Randomized controlled trial of three doses of letrozole for ovulation induction in patients with unexplained infertility. Reproductive Biomedicine Online. 14(5):559-62, 2007 May.

[15] Plourde PV, Dyroff M, Dowsett M, Demers L, Yates R, Webster A. Arimidex ${ }^{\mathrm{TM}}$ : a new oral, once-a-day aromatase inhibitor. J Ster Biochem Mol Biol 1995;53:175-9.

[16] Bisagni G, Cocconi G, Scaglione F, Fraschini F, Pfister CH, Trunet PF. Letrozole, a new oral non-steroidal aromatase inhibitor in treating postmenopausal patients with advanced breast cancer. A pilot study. Ann Oncol 1996;7:99-102.

[17] Bajetta E, Zilembo N, Bichisao E et al. Tumor response and estrogen suppression in breast cancer patients treated with aromatase inhibitors. Ann Oncol 2000;11:1017-22.

[18] Albrecht ED, Aberdeen GW, Pepe GJ. The role of estrogen in the maintenance of primate pregnancy. Am J Obstet Gynecol 2000;182:432-8.

[19] Shi L, Shi SQ, Given RL, von Hertzen H, Garfield RE. Synergistic effects of antiprogestins and iNOS or aromatase inhibitors on establishment and maintenance of pregnancy. Steroids 2003;68:1077-84.

[20] Knudsen UB. First trimester abortion with mifepristone and vaginal misoprostol. Contraception 2001;63:247-50.

[21] Ngai SW, Tang OS, Chan YM, Ho PC. Vaginal misoprostol alone for medical abortion up to 9 weeks of gestation: efficacy and acceptability. Hum Reprod 2000;15:1159-62.

[22] Jain JK, Dutton C, Harwood B, Meckstroth KR, Mishell Jr DR. A prospective randomized, double-blinded, placebo-controlled trial comparing mifepristone and vaginal misoprostol to vaginal misoprostol alone for elective termination of early pregnancy. Human reproduction. 2002;17(6):1477-82.

[23] Kragie L. Aromatase in primate pregnancy: a review. Endoc Res 2002;28:121-8. 
Table 1. Demographic data

\begin{tabular}{|lcccc|}
\hline Demographic data & \multicolumn{2}{c}{$\begin{array}{c}\text { Letrozole and misoprostol regimen } \\
(\mathbf{n = 2 0 )}\end{array}$} & \multicolumn{3}{c|}{$\begin{array}{c}\text { Letrozole and mifepristone } \\
\text { regimen (n=21) }\end{array}$} \\
& Mean (median) & Range & Mean (median) & Range \\
\hline Age (years) & $31.2(32.5)$ & $18-42$ & $30.0(31.0)$ & $19-40$ \\
Height (cm) & $159.0(158.5)$ & $150-167$ & $159.7(160.0)$ & $152-173$ \\
Weight (kg) & $55.6(55.0)$ & $46-68$ & $52.3(53.0)$ & $37-62$ \\
Gestation age (days) & $46.3(43.5)$ & $42-59$ & $48.3(45.0)$ & $42-61$ \\
\hline
\end{tabular}


Table 2. Outcome measures

\begin{tabular}{|c|c|c|c|c|}
\hline \multirow[t]{2}{*}{ Outcome measures } & \multicolumn{2}{|c|}{$\begin{array}{l}\text { Letrozole and misoprostol } \\
\text { regimen }(n=20)\end{array}$} & \multicolumn{2}{|c|}{$\begin{array}{c}\text { Letrozole and mifepristone } \\
\text { regimen }(n=21)\end{array}$} \\
\hline & $\begin{array}{c}\text { Mean } \\
\text { (median) }\end{array}$ & Range & $\begin{array}{c}\text { Mean } \\
\text { (median) }\end{array}$ & Range \\
\hline $\begin{array}{l}\text { Interval between } 1^{\text {st }} \text { dose letrozole } \\
\text { and vaginal bleeding (h) }\end{array}$ & $48.2(51.0)$ & $26.6-55.4$ & $67.7(69.6)$ & $29.2-103.3$ \\
\hline $\begin{array}{l}\text { Interval between targeted drug* } \\
\text { administration and POG passed (h) }\end{array}$ & $9.1(7.9)$ & $2.7-23.6$ & $90.1(93.4)$ & $66.0-121.2$ \\
\hline \multicolumn{4}{|l|}{ Outcome according to protocol } & $5-66$ \\
\hline \multirow[t]{2}{*}{ Complete abortion } & $16 / 20(80 \%)$ & 95\%CI: & $15 / 21(71.4 \%)$ & 95\%CI: \\
\hline & & $56.3-94.3 \%$ & & $47.8-88.7 \%$ \\
\hline \multirow[t]{2}{*}{ Ongoing pregnancy } & $4 / 20(20 \%)$ & 95\%CI: & $5 / 21(23.8 \%)$ & 95\%CI: \\
\hline & & $5.7-43.7 \%$ & & $8.2-47.2 \%$ \\
\hline
\end{tabular}

*Targeted drug: 1 . letrozole and misoprostol = misoprostol, 2. letrozole and mifepristone $=$ mifepristone.

POG - product of gestation. 
Table 3. Side effects profile

\begin{tabular}{|c|c|c|c|c|c|c|c|}
\hline \multirow{2}{*}{$\begin{array}{l}\text { Side } \\
\text { effects }\end{array}$} & \multicolumn{4}{|c|}{ Letrozole + misoprostol $(n=20)$} & \multicolumn{3}{|c|}{ Letrozole + mifepristone $(n=21)$} \\
\hline & $\begin{array}{c}\text { During } \\
\text { pregnancy }\end{array}$ & Day 1-3 & $\begin{array}{c}\text { During } \\
\text { misoprostol }\end{array}$ & Day 3-7 & $\begin{array}{c}\text { During } \\
\text { pregnancy }\end{array}$ & Day1-3 & $\begin{array}{l}\text { Day3-7 } \\
(\mathrm{n}=19)^{*}\end{array}$ \\
\hline Nausea & $7(35 \%)$ & $5(25 \%)$ & $7(35 \%)$ & $6(30 \%)$ & 9 (42.9\%) & $9(42.9 \%)$ & $7(36.8 \%)$ \\
\hline Vomiting & $2(10 \%)$ & $1(5 \%)$ & $1(5 \%)$ & $2(10 \%)$ & 3 (14.3\%) & $4(19.0 \%)$ & 3 (15.8\%) \\
\hline Diarrhea & - & $1(5 \%)$ & - & - & - & - & $1(5.3 \%)$ \\
\hline Fatigue & $3(15 \%)$ & $6(30 \%)$ & $1(5 \%)$ & $9(45 \%)$ & $3(14.3 \%)$ & $6(28.6 \%)$ & $8(42.1 \%)$ \\
\hline Dizziness & $7(35 \%)$ & $3(15 \%)$ & $2(10 \%)$ & $5(25 \%)$ & $3(14.3 \%)$ & $7(33.3 \%)$ & $5(26.3 \%)$ \\
\hline Fainting & - & - & - & - & - & - & - \\
\hline Headache & - & - & $3(15 \%)$ & $4(20 \%)$ & - & $4(19.0 \%)$ & $4(21.1 \%)$ \\
\hline $\begin{array}{l}\text { Lower } \\
\text { abd pain }\end{array}$ & $4(20 \%)$ & $3(15 \%)$ & $13(65 \%)$ & $\begin{array}{c}15 \\
(75 \%)\end{array}$ & $2(9.5 \%)$ & $8(38.1 \%)$ & $\begin{array}{c}16 \\
(84.2 \%)\end{array}$ \\
\hline $\begin{array}{l}\text { Breast } \\
\text { tenderness }\end{array}$ & $3(15 \%)$ & $1(5 \%)$ & - & - & $9(42.9 \%)$ & $2(9.5 \%)$ & $1(5.3 \%)$ \\
\hline Chills & $1(5 \%)$ & - & $3(15 \%)$ & & - & $1(4.8 \%)$ & - \\
\hline Rash & - & -- & - & $1(5 \%)$ & & & \\
\hline Fever $\S$ & - & -- & $1(5 \%)$ & - & - & - & - \\
\hline Others & & & & & - & - & $\begin{array}{c}1 \% \\
(5.3 \%)\end{array}$ \\
\hline
\end{tabular}

* One subject withdrawn from study as she had surgical evacuation in private hospital because of personal reason; another subject underwent suction evacuation and laparoscopic right ovarian cystectomy due to ovarian cyst complication while she had complete abortion before operation. \# Lower abdominal pain.

$\S$ Fever $\geq 38^{\circ} \mathrm{C}$.

* Low back pain. 
Fig 1. Hormonal profile of letrozole and misoprostol regimen. (All values of hormonal concentration are presented as median.)

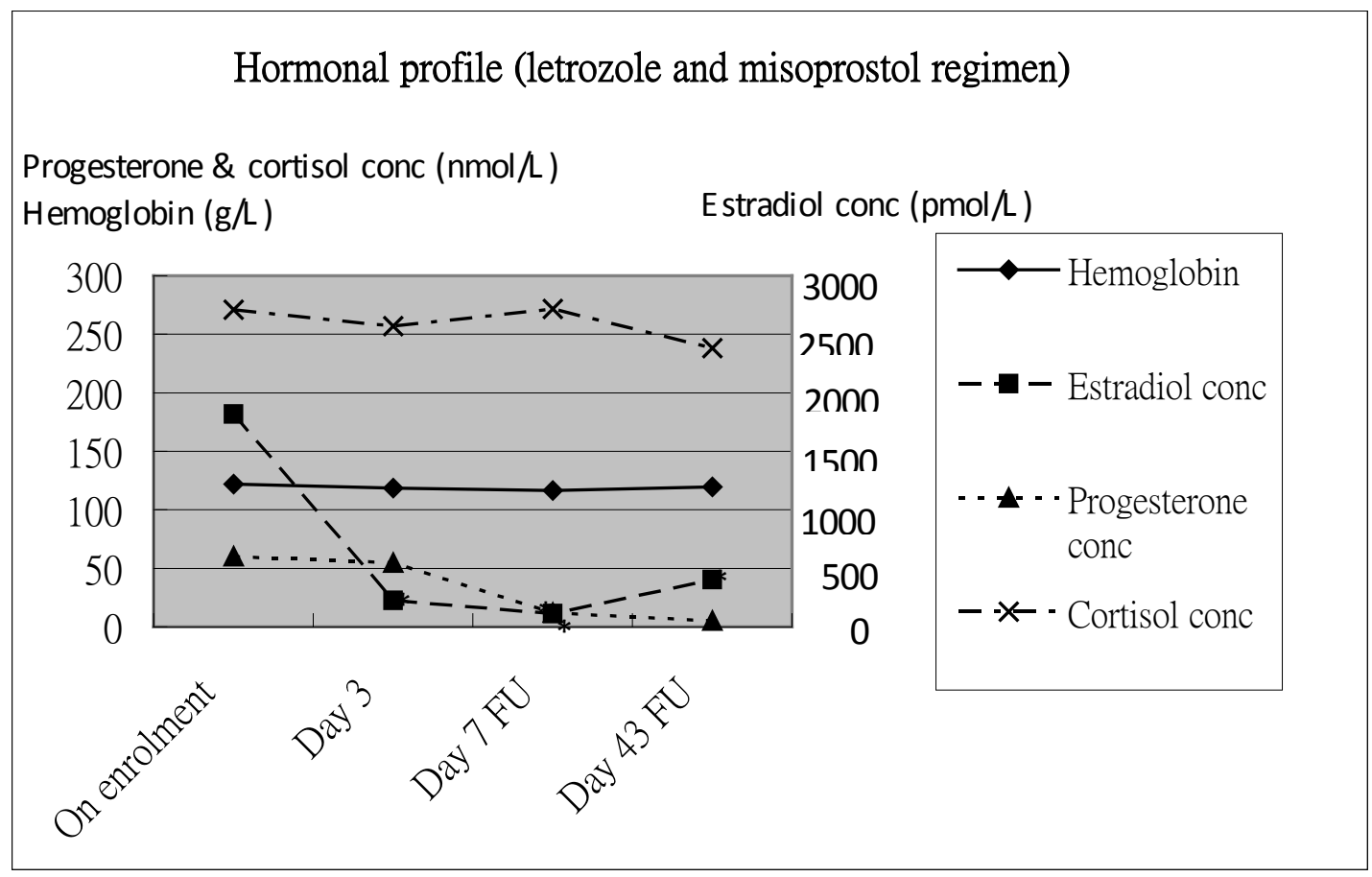

Datum with an asterisk $(*)$ showed a $p$-value of statistical significance comparing with the datum before it. (for example, D7 datum compared with D3 datum) 
Fig 2. Hormonal profile of letrozole and mifepristone regimen. (All values of hormonal concentration are presented as median.)

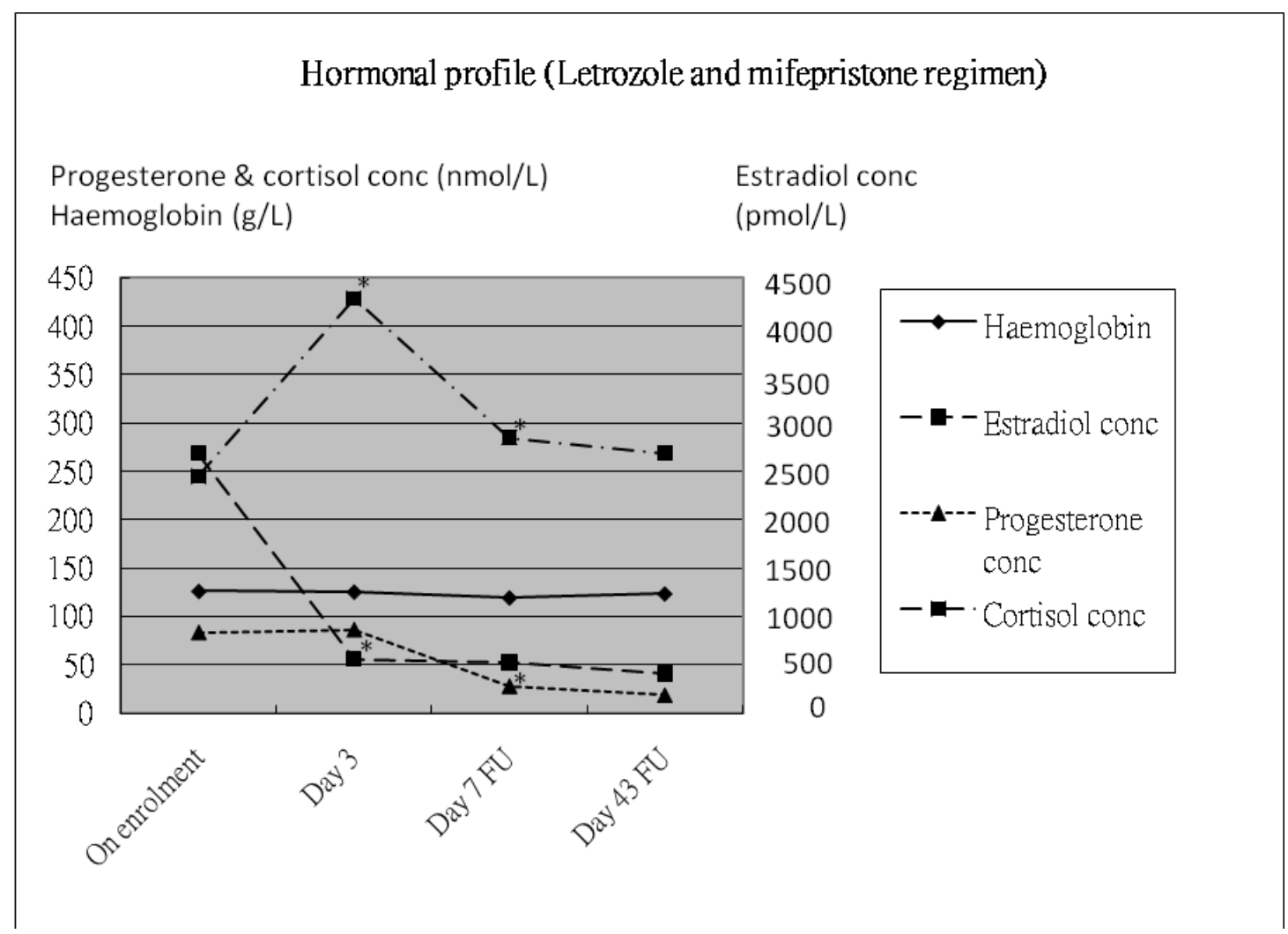

Datum with an asterisk $(*)$ showed a $p$-value of statistical significance comparing with the datum before it. (for example, D7 datum compared with D3 datum) 